\title{
Effects of Heptanol on Neurogenic Contractions of Vas Deferens: A Comparative Study of Stimulation Frequency in Guinea Pig and Rat
}

\author{
D. PALANI and R. MANCHANDA \\ Biomedical Engineering Group, School of Bioscience and Bioengineering, Indian Institute of Technology-Bombay, Mumbai, 400076, India
}

\begin{abstract}
This study examines the role of gap junctional communication in smooth muscle in relation to the frequency of stimulation and the innervation density of the tissue in the generation of neurogenic contractions. Toward this end the effects of heptanol, a gap junctional blocker, on the neurogenic contractions of guinea pig and rat vas deferens at different frequencies of stimulation (single pulse, 5, 10, 20,40,60, and $80 \mathrm{~Hz}$ ) were studied. In both the prostatic and epididymal halves of these tissues, heptanol abolished the neurogenic contractions at the lower frequencies of stimulation. At higher frequencies, contractions
\end{abstract}

were resistant to heptanol action. The effect of heptanol on the neurogenic contractions was found to decrease with increasing stimulation frequency. The neurogenic contractions of rat vas deferens were more resistant to heptanol than those of guinea pig vas deferens. Our data indicate that gap junctional communication is significant in the generation of neurogenic contractions in both guinea pig and rat vas deferens in a frequency-dependent manner, and we discuss the mechanisms underlying these findings.

Key words: rodent vas deferens, gap junction, heptanol, neurogenic contractions, smooth muscle.

n smooth muscle organs like the mammalian vas deferens, direct neuronal control of the muscle cells is limited by factors such as sparse innervation of tissue, low probability of neurotransmitter release, and the variable width of the synaptic cleft [1-3]. Since the number of muscle cells receiving direct neuronal input is low, intercellular communication between cells is thought to be of vital importance in the coordinated functioning of the organ. Gap junctions have been shown to form direct connections between cells giving rise to cell-to-cell coupling [4] and are thought to mediate the uniform spread of biochemical and electrical signals through the tissue to enable coordinated action [5]. The role of gap junctions in the coordinated contractility of the vas deferens is supported by experiments conducted in the presence of heptanol, a relatively specific gap junction blocker [6].

In both the guinea pig vas deferens (GPVD) and rat vas deferens (RVD), it has been shown that a denervation of the tissue increases the extent of cell-to-cell coupling [7]. Similarly, it is thought that the contribution of intercellular coupling via gap junctions in the spread of signals across the tissue could vary, depending on factors such as the pattern of innervation, frequency of nerve stimulation, and the extent of cell-to-cell coupling. For example, since it is known that the density of innervation and the number of close contact varicosities in RVD is higher than that found in GPVD [1, 8], it may be predicted that in GPVD the physiological response of the tissue would depend on intercellular communication via gap junctions to a greater extent in comparison with RVD, where a greater proportion of cells receive direct innervation. Similarly, the frequency of stimulation used to elicit contractions could also be a factor significantly modifying the extent of gap junctional contribution to the tissue's contractile response. It has been shown that higher frequencies of nerve stimulation result in the facilitation and recruitment of neurotransmitter release sites [9-11]; this in turn would directly activate a larger fraction of smooth muscle cells in the tissue. Even though studies have been conducted previously to assess the role of gap junctions in several other smooth muscle tissues, the relative significance of gap junctions as a function of frequency of stimulation has not yet been reported in any smooth muscle system.

In the present report, we have studied neurogenic contractions in two models, the RVD and the GPVD, systems closely related to each other $[12,13]$ but significantly different in their innervation patterns $[1,8,14,15]$, to test these predictions. The relative contribution of gap junctions to the neurogenic contractions in these systems was investigated at different frequencies of stimulation, using the relatively specific gap junctional blocker 1-heptanol [16-18]. We have taken neurogenic contractions to serve as an index of coordination because this would directly reflect the amount of coordination achieved by the tissue at varying levels of cell-to-cell coupling at different frequencies of stimulation. Since it is known that two halves of

Received on Nov 10, 2005; accepted on Dec 13, 2005; released online on Dec 18, 2005; DOI: 10.2170/jjphysiol.RP001205 Correspondence should be addressed to: Rohit Manchanda, Biomedical Engineering Group, School of Bioscience and Bioengineering, Indian Institute of Technology-Bombay, Mumbai, 400076, India. Tel: +91-22-25767765. E-mail: rmanch@iitb.ac.in 
the vas differ notably in their neurogenic contractility, e.g., in having markedly different components mediated by adrenergic and purinergic components (the purinergic component being predominant in the prostatic half and the adrenergic in the epididymal half), the possibility of regional variation in the role of cell-to-cell communication was also investigated by studying the responses of the prostatic and epididymal halves of the vas separately.

\section{METHODS}

Animals. Male Wistar rats $(250-350 \mathrm{gm})$ and male Dunkin-Hartley guinea pigs (450-600 gm) obtained from the National Institute of Nutrition, Hyderabad, were used in this study. The experimental procedures were approved by the Animal Experimentation Ethics Committee of Indian Institute of Technology, Bombay.

Chemicals. Salts used in Krebs solution and 1-heptanol were of analytical grade (S.D. Fine Chemicals Ltd., Mumbai, India). Noradrenaline (Arterenol bitartarate) and prazosin were obtained from Sigma-Aldrich, Mumbai, India, and Suramin from Tocris-Cookson Ltd., UK.

Contraction studies. The animals were sacrificed by stunning and exsanguination. Vasa deferentia were carefully dissected out and the adhering connective tissue removed. The organ was divided equally into two pieces, prostatic and epididymal halves. The tissue pieces were passed through $\mathrm{Ag} / \mathrm{AgCl}$ electrodes (positioned at the prostatic end) and mounted vertically inside the organ bath ( $25 \mathrm{ml}$ capacity). The prostatic end of the tissue was attached to a force transducer (Recorders \& Medicare Systems, Chandigarh, India), whereas the epididymal end of the tissue was affixed firmly fixed to the bottom of the organ bath to record isometric contractions. The tissue was continuously superfused with Krebs solution $(\mathrm{NaCl} 118.4$ $\mathrm{mM}, \mathrm{KCl} 4.7 \mathrm{mM}, \mathrm{MgCl}_{2} 1.2 \mathrm{mM}, \mathrm{CaCl}_{2} 2.5 \mathrm{mM}$, $\mathrm{NaHCO}_{3}, 25.0 \mathrm{mM}, \mathrm{NaH}_{2} \mathrm{PO}_{4} 0.4 \mathrm{mM}$, and Glucose 11.1 $\mathrm{mM})$ bubbled with carbogen $\left(95 \% \mathrm{O}_{2}\right.$ and $\left.5 \% \mathrm{CO}_{2}\right)$ to maintain the $\mathrm{pH}$ at 7.4. All experiments were conducted at room temperature $\left(25-30^{\circ} \mathrm{C}\right)$. The tissues were incubated with heptanol at the stated concentrations for 4 min before stimulation.

Supramaximal tetanic field stimulation was carried out using the following rectangular voltage pulses (Amplitude, Pulse Width): 70-80 V and 0.5-1 ms for neurogenic stimulation and $5 \mathrm{~V}$ and $200-250 \mathrm{~ms}$ for myogenic stimulation $(<1 \mathrm{~Hz})$, delivered by an electronic stimulator (DS2A, Digitimer, UK). Seven different stimulation protocols were used for neurogenic stimulation, namely, single pulse, $5,10,20,40,60$, and $80 \mathrm{~Hz}$ with a $25 \mathrm{~min}$ time interval between successive stimulations. Signals obtained from the force transducer were amplified, and the output of the amplifier was fed to a digital tape recorder (DTR1204, Biologic, France).

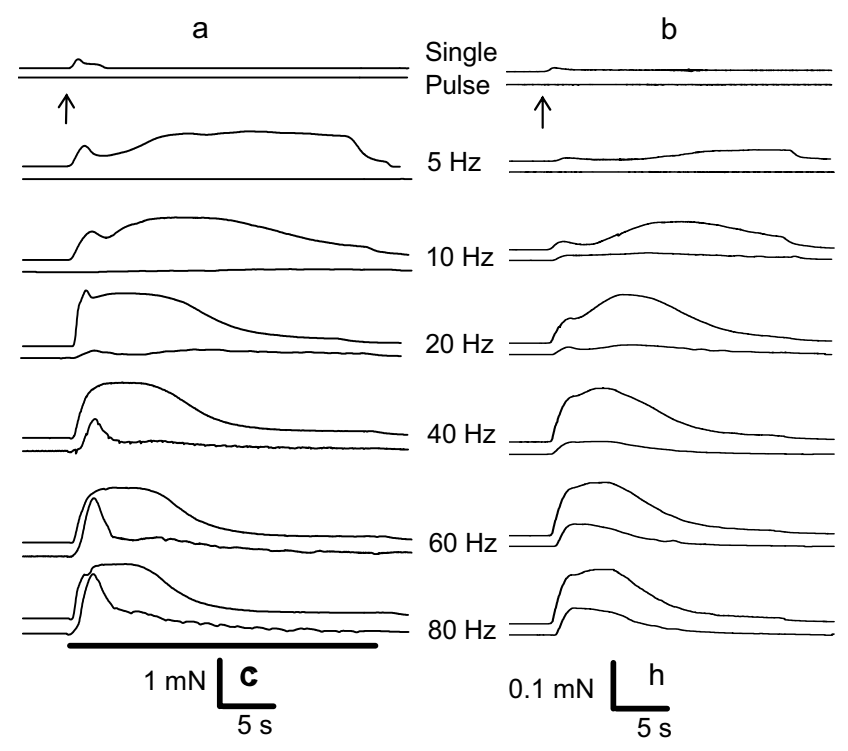

Fig. 1. Effect of $2 \mathrm{mM}$ heptanol on neurogenic contractions of the (a) prostatic and (b) epididymal halves of guinea pig vas deferens in a single tissue at the stimulation frequency indicated. In each set of traces, the upper one is the control and the lower one the response in the presence of $2 \mathrm{mM}$ heptanol. The duration of stimulation (30 s) for the tetanic stimulation is marked below the curves (thick bar). For single pulse stimulation, the arrow below the curve marks the point of stimulation. Note that the scale for traces obtained in the presence of heptanol $(\mathrm{h})$ is 10 times smaller in magnitude compared with the control (c) traces, as indicated in the bottom of the figure.

Statistics. An unpaired Student's $t$-test was used as a test for statistical significance between the sets of results. $P<0.05$ was taken as statistically significant. Data have been reported as mean $\pm \mathrm{SD}$.

\section{RESULTS}

Tetanic field stimulation of both the GPVD and RVD resulted in a characteristic neurogenic biphasic contractile response (see Figs. 1 and 2, control traces) with physiological and pharmacological characteristics as reported previously $[19,20]$, e.g., in terms of the relative amplitudes of the two phases in the prostatic and epididymal halves [21] (Figs. 1 and 2) and their sensitivity to the purinergic and noradrenergic receptor blockers, suramin and prazosin $[21,22]$. Below we present the effects of heptanol on the frequency-dependent contractile response of the prostatic and epididymal halves of the GPVD and RVD. We have used heptanol at a concentration of $2 \mathrm{mM}$ throughout our investigation because this concentration is known to be adequate for its uncoupling effects to be clearly observed [6] while still being low enough to ensure specificity of action [23]. 


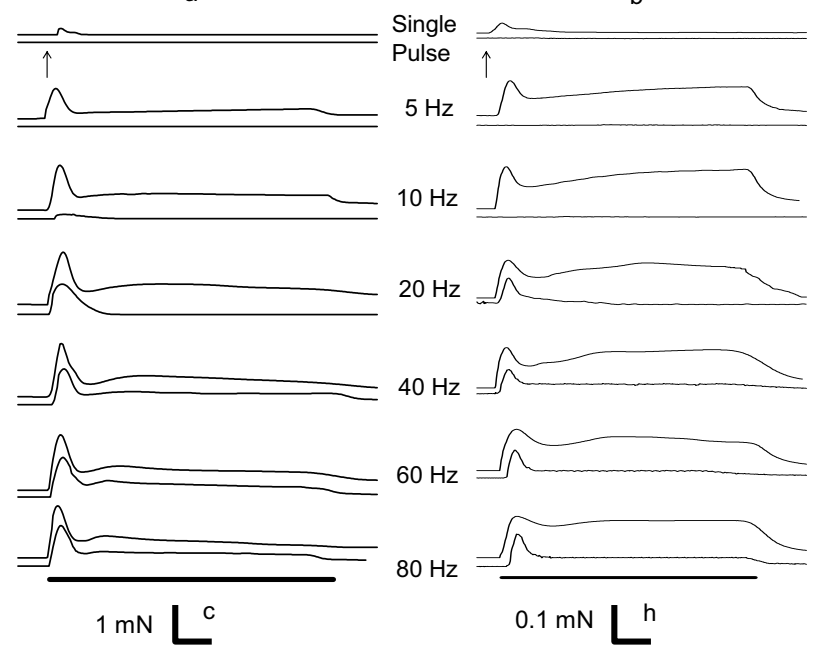

Fig. 2. Effect of $2 \mathrm{mM}$ heptanol on neurogenic contractions of the (a) prostatic and (b) epididymal halves of rat vas deferens in a single tissue at single pulse, $5,10,20,40,60$, and $80 \mathrm{~Hz}$ of stimulation. In each set of traces, the upper one is the control and the lower one the response in the presence of $2 \mathrm{mM}$ heptanol. The duration of stimulation (30 s) for the tetanic stimulation is marked below the curves. For single pulse stimulation, the arrow below the curve marks the point of stimulation. Note that the scale for traces obtained in the presence of heptanol $(\mathrm{h})$ is 10 times smaller in magnitude compared with the control (c) traces, as indicated in the bottom of the figure.

\section{GPVD}

Figure 1 shows representative traces illustrating the effect of $2 \mathrm{mM}$ heptanol on neurogenic contractions of GPVD in the prostatic half (left panel) and epididymal half (right panel). In the prostatic half(Fig. 1a), 2 mM heptanol completely abolished the neurogenic contractions evoked by single pulse or $5 \mathrm{~Hz}$ stimulation $(n=7)$. A heptanol-resistant component was observable at stimulation frequencies of $10 \mathrm{~Hz}$ and above in both phases. As can be seen in Fig. 3, which displays the percentages of the resistant components at different frequencies, the amplitude of the heptanol-resistant component in both phases increased with an increase in stimulation frequency (Fig. 3a), ranging from $1 \%$ to $13 \%$ of the control contraction at 10 and $80 \mathrm{~Hz}$, respectively. When the two phases of the contraction were compared, it was found that the percentage of the heptanol-resistant component in the first phase was higher than that in the second phase at higher stimulation frequencies (40,60, and $80 \mathrm{~Hz} ; P<0.05, n=7$, see Fig. 1), but not at 10 and $20 \mathrm{~Hz}$.

In the epididymal half, unlike the prostatic half, contractions elicited at $5 \mathrm{~Hz}$ were resistant to heptanol, though this component was on the order of only $\sim 2 \%-4 \%$ of the control contraction (not observable in Fig. 1b, see Fig. 3b). The frequency dependence of the heptanol-resistant contraction showed a similar trend to that in the prostatic half; i.e., the amplitude of the heptanol-resistant component of both phases increased with an increase in stimulation frequency (Fig. 3b). On comparing the two phases of contraction, the first phase was found to have a higher percentage of the heptanol-resistant component than the second phase at higher stimulation frequencies $(40-80 \mathrm{~Hz} ; P<0.05, n=7$; asterisks in Fig. 1 b), but not at lower ones $(5-20 \mathrm{~Hz} ; P>0.05, n=7)$. These findings are qualitatively similar to those for the prostatic half; however, there were quantitative differences between the responses of the two halves. For example, both phases of the epididymal half were more resistant to heptanol than the prostatic half at all frequencies of stimulation $(P<0.05, n$ $=7$ ) except the highest $(60-80 \mathrm{~Hz}$; data not illustrated).

\section{RVD}

In the prostatic half of the RVD, $2 \mathrm{mM}$ heptanol completely abolished the neurogenic contractions elicited by single pulse and $5 \mathrm{~Hz}$ stimulation $(P<0.05 ; n=7)$ (Fig. 2a). A heptanol-resistant contraction was observed in the
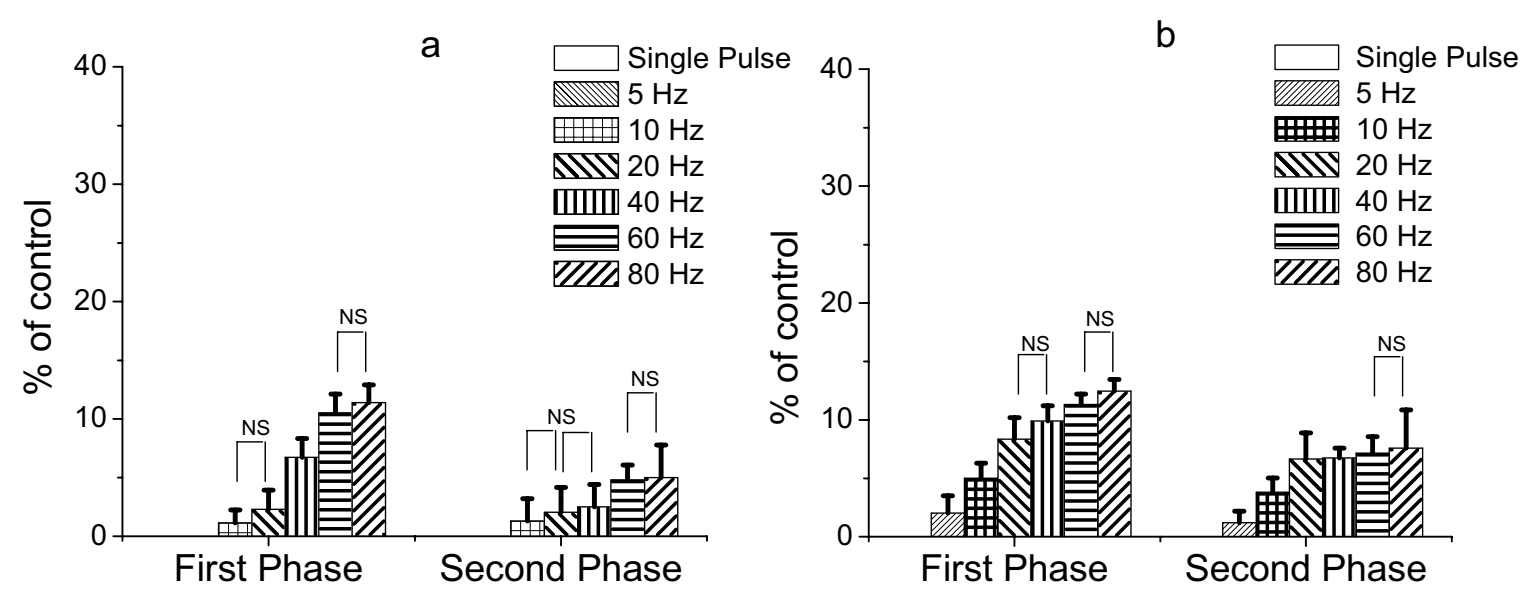

Fig. 3. Effect of heptanol on neurogenic contractions of GPVD at different frequencies of stimulation, (a) prostatic and (b) epididymal half. The increasing frequency of stimulation significantly increased the amplitude of the heptanol resistant phase $(P<$ $0.05, n=7$ ) (except between the frequencies indicated as NS). 


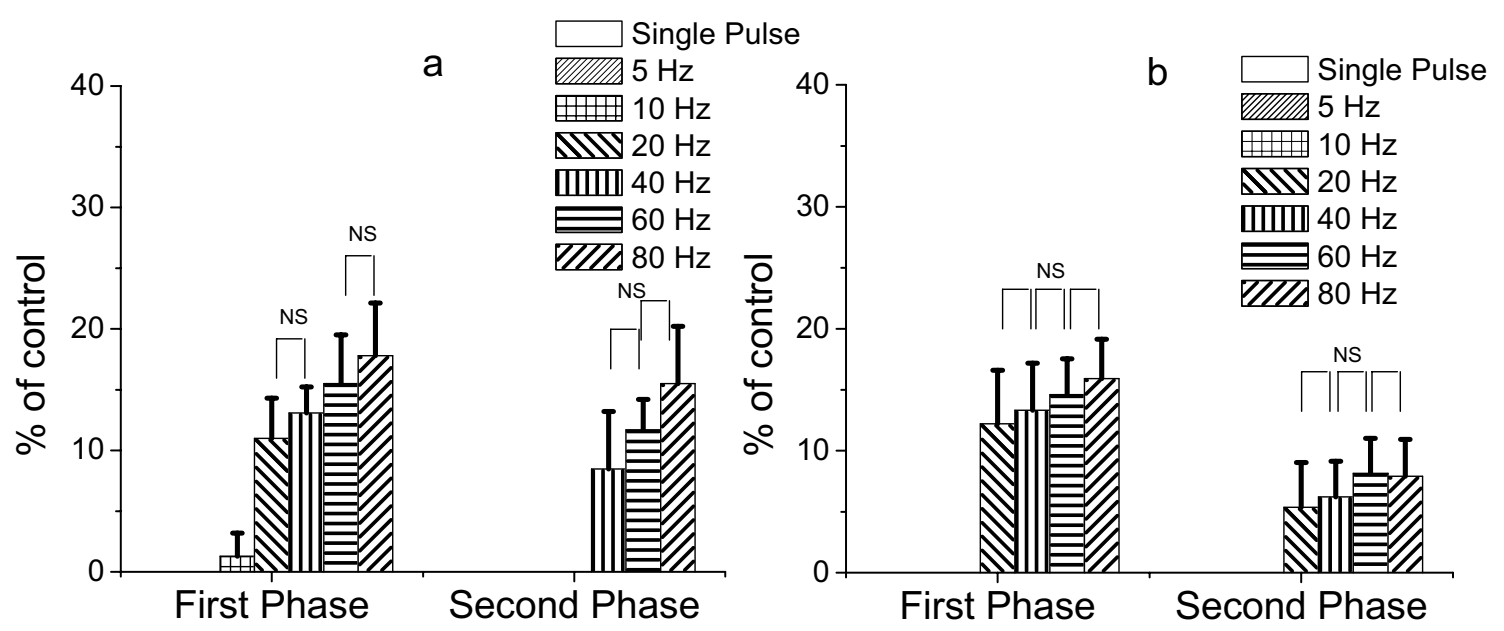

Fig. 4. Effect of heptanol on neurogenic contractions of RVD at different frequencies of stimulation, (a) prostatic and (b) epididymal half. The increasing frequency of stimulation significantly increased the amplitude of the heptanol-resistant phase $(P<$ $0.05, n=7$ ) (except between the frequencies indicated as NS).

first phase at $10 \mathrm{~Hz}$ stimulation $(1.3 \pm 1.9 \%$ of control contraction; $n=7$ ), but at $20 \mathrm{~Hz}$ both phases were resistant. The first phase of the heptanol-resistant component increased with the increasing frequency of stimulation $(20-80 \mathrm{~Hz})$ from $1 \%$ to $16 \%(n=7)$ (Fig. $4 a)$. In the second phase, an increase in the stimulation frequency did not significantly affect the amplitude of the heptanol-resistant component $(P>0.05 ; n=7)$ (Fig. 4a). As observed in GPVD, this component was more prominent in the first phase of the contraction than in the second phase except at 60 and $80 \mathrm{~Hz}$ of stimulation $(P<0.05 ; n=7)$.

In the epididymal half of the RVD, heptanol completely abolished both phases of neurogenic contractions elicited with single pulse and with $5 \mathrm{~Hz}$ and $10 \mathrm{~Hz}$ stimulation (Fig. 2b). The heptanol-resistant components at 20, 40, 60 , and $80 \mathrm{~Hz}$ were not significantly different from each other in either phase of the contraction. Of the two phases, the first was more resistant to heptanol than the second at all four frequencies $(P<0.05 ; n=7)$ (Fig. 4b). There was no significant difference in the amplitude of the first phase of the heptanol-resistant component between the prostatic and epididymal halves of the RVD. However the second phase of the heptanol-resistant component was significantly higher in the prostatic half than the epididymal half at stimulation frequencies of 60 and $80 \mathrm{~Hz}$.

\section{Comparison: GPVD vs. RVD}

Figure 5 shows a comparison of the percentage of the heptanol-resistant component observed in GPVD and RVD for the prostatic half of the tissue (Fig. 5a) and the epididymal half (Fig. 5b). The data are plotted as the difference between the percentage of heptanol-resistant component observed in RVD and GPVD (RVD-GPVD). From Fig. 5 it is seen that in general, the heptanol-resistant component in RVD was higher compared to that of the GPVD, particularly in the range $20-80 \mathrm{~Hz}$, this difference

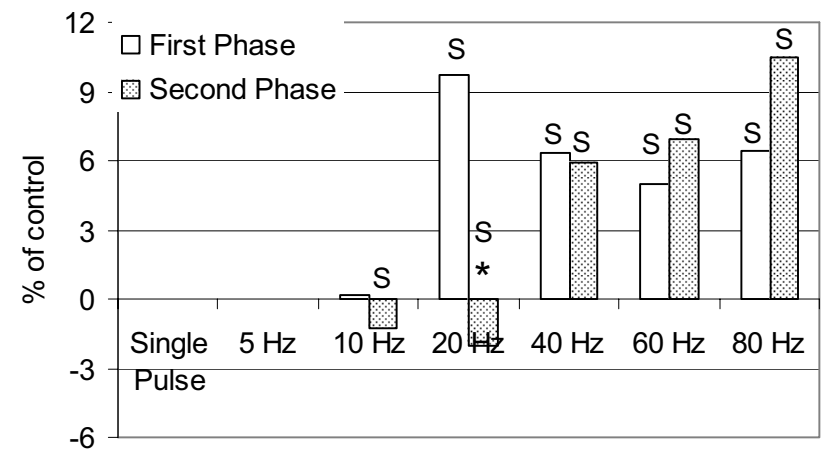

b

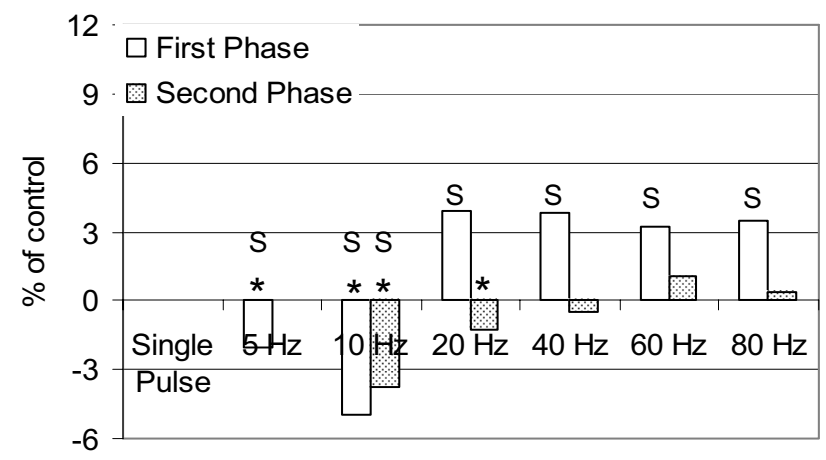

Fig. 5. Comparison of heptanol-resistant component observed between RVD and GPVD. (a) In prostatic and (b) epididymal halves. The comparison is represented as the difference between the component observed in RVD and GPVD, i.e., RVD-GPVD. S indicates the significant difference between RVD and GPVD at $P<0.05$. * indicates that the heptanol-resistant component was absent in RVD and present only in GPVD.

being more prominent for the prostatic half $(n=7 ; P<$ 0.05 ) (Fig. 5a). In the epididymal half, the difference was more variable. For instance, the first phase was higher for RVD over the range of frequencies $20-80 \mathrm{~Hz}$, but signifi- 

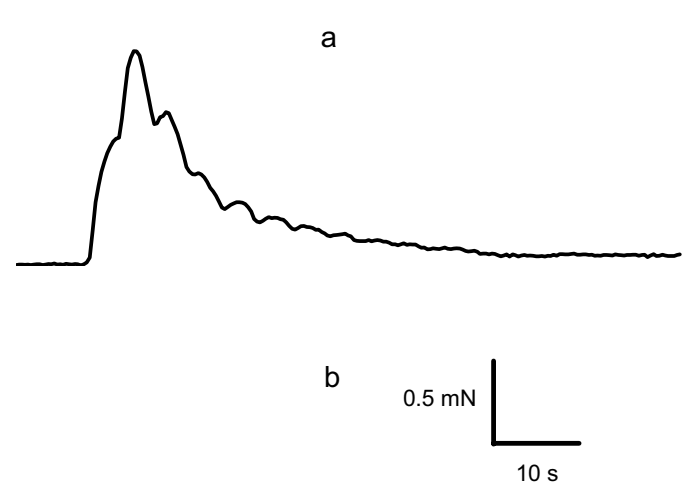

Fig. 6. Effect of $2 \mathrm{~mm}$ heptanol on myogenic contraction vas deferens. (a) Control. (b) In the presence of heptanol. The thick line represents the duration of stimulation for both traces. In the myogenic control contractions, a peak is observed for each stimulation pulse delivered. Because the stimulation frequency used was $0.33 \mathrm{~Hz}$, a separate peak is seen at approximately every $3 \mathrm{~s}$.

cantly lower at 5 and $10 \mathrm{~Hz}(n=7 ; P<0.05)$ (Fig. $5 b)$. An interesting observation was that the second phase of the epididymal half consistently showed no significant difference between RVD and GPVD over the frequency range 20-80 Hz (Fig. 5b).

\section{Effect of heptanol on myogenic contractions}

Myogenic contractions were elicited using long-duration, low-amplitude stimulus pulses, which are known to excite smooth muscle directly [24]. The possibility of any neurogenic contribution with the stimulus protocol used was eliminated because the myogenic contractions were not affected in the presence of $300 \mu \mathrm{M}$ suramin and $10 \mu \mathrm{M}$ prazosin (blockers of the purinergic and adrenergic neurogenic components, respectively) [6]. Heptanol $(2 \mu \mathrm{M})$ completely abolished the myogenic contractions evoked in the presence of prazosin and suramin in GPVD and RVD (Fig. 6) in both halves of the tissue $(n=7)$.

\section{DISCUSSION}

To explore the contribution of cell-to-cell coupling in the neurogenic contractility of syncytial smooth muscle we have used heptanol, the relatively specific gap junction blocker, to gauge the significance of gap junctional communication in the generation of contractions at different frequencies of stimulation in two models reported to differ in their innervation density, i.e., RVD and GPVD. We used heptanol at a concentration of $2 \mathrm{mM}$ for this purpose, since a wide range of approaches, including single-channel conductance measurement, $\mathrm{Ca}^{2+}$ imaging, and contraction studies, have demonstrated that heptanol is a specific and selective gap junctional blocker in smooth muscles at this concentration $[4,16,23,25,26]$. Below, we discuss our observations with respect to frequency of stimulation before moving on to a comparison of GPVD and RVD and the two phases of the contractions.

Effect of frequency of stimulation. A salient feature of our results is the frequency dependence of the heptanol-resistant component of contractions in the rodent vas deferens. With single-pulse stimulation, $2 \mathrm{~mm}$ heptanol abolished both phases of the neurogenic contraction in both halves of GPVD and RVD (Figs. 1 and 2). This abolition suggests that a cell-to-cell spread of signals might play a dominant role in generating contractions produced by single stimuli. In contrast, at higher frequencies of stimulation a heptanol-resistant component emerged, its amplitude increasing with the increasing frequency of stimulation (Figs. 3 and 4). We advance a possible explanation of these findings in terms of gap junctional contribution to neurogenic contractions as follows. At lower frequencies of stimulation, a combination of factors, such as the normally low probability of stimulation-evoked neurotransmitter release from autonomic varicosities $(<0.01$ in GPVD; [27]; $\sim 0.02$ in mouse vas deferens, a system similar to RVD [28]) and an innervation pattern, where not every smooth muscle cell is directly innervated (especially in GPVD [1]), reduces the likelihood of individual smooth muscle cells receiving direct neuronal input. Thus the recruitment of cells for contraction at lowstimulation frequencies might rely more heavily on the spread of excitation via gap junctions, an idea that is supported by the complete or almost complete abolition of contractions at the lowest frequencies (single pulse stimulation and $5 \mathrm{~Hz}$ ) in both GPVD and RVD. In contrast, at higher frequencies of stimulation the frequency-dependent facilitation of neurotransmitter release known to occur at these neuroeffector junctions $[28,29]$ can result in an increase both in the probability of per-stimulus release from an individual varicosity as well as an increase in the average number of varicosities releasing neurotransmitters across the ground plexus for each stimulus. Thus at higher stimulation frequencies, the proportion of directly activated cells may increase significantly, and the recruitment of cells for contraction may rely less on a gap junction mediated spread of excitation. Our observation that the heptanol-resistant contraction increases at higher stimulation frequencies in both GPVD and RVD and in both halves of the tissues is consistent with this prediction.

A further significant consideration in this regard is the involvement of both close-contact varicosities (CCVs; separation 10-40 $\mathrm{nm}$ ) and loose-contact varicosities (LCVs; separation 100-200 nm) in mediating neurotransmission in the vas deferens [30]. In particular, it has been shown that at low frequencies of activation, a neurotransmitter released from LCVs may act only weakly on the smooth muscle cells because of its diffusion away from the wide synaptic cleft. If the amount of transmitter released from LCVs is also subject to facilitation in a fre- 
quency-dependent manner, at higher frequencies the postjunctional action of the neurotransmitters would then be increased, again leading to a greater proportion of directly activated cells and a higher amplitude of the heptanol resistant contraction [30,31].

Another general feature of the heptanol-resistant component in both species was that it was not significantly different from $60 \mathrm{~Hz}$ to $80 \mathrm{~Hz}$. This may indicate a "saturation" effect, most likely of the frequency-dependent probability of neurotransmitter release from each varicosity, and consequently of the mean number of varicosities activated across the ground plexus per stimulus.

Comparison between GPVD and RVD. Gap junctional communication has been reported previously to play a profound role in determining the characteristics of neurogenic contractions in GPVD elicited at a stimulation frequency of $8 \mathrm{~Hz}$ [6]; our present results confirm this finding and extend it both over a broad range of frequencies and to RVD. A second key aspect of our results is that at most frequencies of stimulation, the component of contraction resistant to heptanol was significantly higher in RVD than in GPVD in both halves of the organ (Fig. 5). A reasonable explanation for this observation may lie in the higher density of innervation reported in RVD compared with GPVD $[1,14]$. These results would therefore appear to suggest a relatively lower contribution of gap junctional communication in the neurogenic contractions of muscles that receive a relatively dense innervation, most likely because of the greater proportion of directly activated cells in the latter. This conclusion is analogous with those on the frequency dependence of the heptanol-resistant contraction, because also in that case, an effective increase in the number of directly activated cells (brought about by frequency-dependent facilitation) appears to reduce the component of contraction mediated by gap junctional communication. It is interesting that the denervation of both GPVD and RVD has been reported to result in an increase in the intercellular electrical coupling of the smooth muscle cells [7]. This would point toward an inherent compensatory mechanism in which cell-to-cell coupling assumes a larger role in mediating contractions when the neuronal input is compromised, a scenario consistent with our inference, and this would be an interesting issue for exploration in future work.

Comparison between first and second phases. In both halves of GPVD, the first phase of the neurogenic contraction was more resistant to heptanol than the second phase at higher frequencies of stimulation. Since the first phase is known to be purely purinergic in nature and the second adrenergic [19], this difference in sensitivity could indicate a difference in the extent of gap junction-mediated contribution to the purinergic and adrenergic components of neurotransmission. Earlier studies on neurogenic contraction of GPVD have shown that the susceptibilities of purinergic and adrenergic components to inhibition by cell-to-cell coupling could be very different (6). Our present observations, however, show that in the RVD such a difference in susceptibility may not exist. This may result because both purinergic and adrenergic components are present in both phases of RVD at significant magnitudes [21].

In terms of the significance of gap junctional communication for the two halves of the vas, we found that in GPVD (but not in RVD) the epididymal half was more resistant to heptanol than the prostatic half in both phases of the heptanol-resistant component. This suggests that in GPVD there may be a greater contribution of gap junctional communication in the prostatic half compared to that of the epididymal half. This could result from either (i) a greater degree of gap junction mediated cell-to-cell coupling in the prostatic half, or (ii) a greater innervation density of the epididymal half compared to the prostatic half, a conclusion supported indirectly by the finding that nitric oxide synthase containing nerve fibers are more numerous in the epididymal half [32].

Our interpretation of the findings reported here rests on the assumption that heptanol is a relatively specific gap junctional blocker, for which there is strong evidence across several tissues and organs [4, 16-18]. Although some studies have reported nonjunctional actions of heptanol in some systems [33-35], heptanol has been shown to block gap junctions in a specific and concentration-dependent manner in systems such as rat aorta, rabbit heart, corpus cavernosum, and hepatocytes [36-39]. In our own studies, concentrations of heptanol up to $2 \mathrm{mM}$ have not been shown to disturb the resting membrane potential in GPVD or RVD ([40]; Palani and Manchanda, unpublished observations), indicating an absence of its action on "background" ionic channels. Further, $2 \mathrm{mM}$ heptanol left unaffected the transient depolarizations produced by the exogenous application of NA, which are known to be caused by the activation of L-type $\mathrm{Ca}^{2+}$ channels via the $\alpha_{1}$-adrenoceptor pathway [41], indicating that heptanol was not acting at a level downstream of $\alpha_{1}$ receptor activation in the NA-mediated cascade. Our results showing the inhibition of myogenic contractions by heptanol strengthen these conclusions. Electrically evoked smooth muscle contraction results from an influx of $\mathrm{Ca}^{2+}$ into the cells through voltage-gated calcium channels [42]. In the present experiments, action potentials during muscle stimulation would be directly evoked in only a narrow band of cells directly encircled by the cathode. A much larger number of cells are presumably recruited for contractions by the spread of the action potentials through gap junctions. If heptanol blocks gap junctional communication, the recruitment of cells for contractions would be reduced, resulting in an inhibition of the myogenic response.

Conclusions. In conclusion, we suggest that gap junction mediated cell-to-cell couplings may have a signifi- 
cant role in facilitating the coordinated contractions of the vas deferens, and that the extent of this contribution could vary with the frequency of stimulation. From our comparison of the effects on RVD and GPVD, it would appear that denser innervation patterns may reduce the dependency of neurogenic contractions on the cell-to-cell spread of signals, in accordance with expectations. Furthermore, gap junctional contribution may vary significantly, depending on the neurotransmitter involved or the region of the muscle studied. Thus a modulation of neurotransmission by gap junctions can vary not only from species to species, but even within a single organ, depending on the frequency of stimulation. These findings might help in the appreciation of the diversity of mechanisms involved in smooth muscle contractility, especially those involving different innervation patterns and different degrees of cell-to-cell coupling.

We acknowledge financial support provided by the Department of Science \& Technology (DST), India (SP/SO/B-11/2000), and by IIT-Bombay under the Cross Disciplinary Research Grant.

\section{REFERENCES}

1. Merrillees NCR. The nervous environment of individual smooth muscle cells of the guinea pig vas deferens. J Cell Biol. 1968;37:794-817.

2. Ward SM, Sanders KM, Hirst GD. Role of interstitial cells of Cajal in neural control of gastrointestinal smooth muscles. Neurogastroenterol Motil. 12004;6:1127.

3. Kobilo T, Szurszewski JH, Farrugia G, Hanani M. Coupling and innervation patterns of interstitial cells of Cajal in the deep muscular plexus of the guinea-pig. Neurogastroenterol Motil. 2003;15:635-41.

4. Hennig GW, Smith CB, O'Shea DM, Smith TK. Patterns of intracellular and intercellular $\mathrm{Ca}^{2+}$ waves in the longitudinal muscle layer of the murine large intestine in vitro. J Physiol. 2002;543:233-53.

5. Haefligera J, Nicoda P, Medab P. Contribution of connexins to the function of the vascular wall. Cardiovas Res. 62004;2:345-56.

6. Venkateswarlu K, Dange SY, Manchanda R. Effect of heptanol on the neurogenic and myogenic contractions of the guinea-pig vas deferens. Br J Pharmacol. 1999;126:227-34

7. Goto K, Westfall DP, Fleming WW. Denervation-induced changes in electrophysiologic parameters of the smooth muscle of the guinea-pig and rat was deferens. J Pharmacol Exp Ther. 1978;204:325-33.

8. Richardson KC. The fine structure of autonomic nerve endings in smooth muscle of the rat vas deferens. J Anat. 1962;96:427-42.

9. Cheung DW. Synaptic transmission in the guinea-pig vas deferens: the role of nerve action potentials. Neuroscience. 1990;37:127-34.

10. Cunnane TC, Searl TJ. Neurotransmitter release mechanisms in autonomic nerve terminals. In: Stjärne L, Greengard P, Grilner S, Hökfelt T, Ottoson D, editors. Molecular and cellular mechanisms of neurotransmitter release. New York: Raven Press; 1994. p. 425-59.

11. Zucker RS. Calcium and transmitter release. J Physiol (Paris). 1993:87:25-36.

12. Allcorn RJ, Cunnane TC, Kirkpatrick K. Actions of alpha, beta-methylene ATP and 6-hydroxydopamine on sympathetic neurotransmission in the vas deferens of the guinea pig, rat and mouse: Support for cotransmission. Br J Pharmacol. 1986;89:647-59.

13. Westfall DP. The effects of denervation, cocaine, 6-hydroxydopamine and reserpine on the characteristics of drug-induced contractions of the depolarized smooth muscle of the rat and guinea-pig vas deferens. J Pharmacol Exp Ther. 1977;201:267-75.

14. Merrillees NC, Burnstock G, Holman M. Correlation of fine structure and physiology of the innervation of smooth muscle in the guinea pig vas deferens. $J$ Cell Biol. 1963;19:529-50.

15. Goto K, Millecchia LL, Westfall DP, Fleming WW. A comparison of the electrical properties and morphological characteristics of the smooth muscle of the rat and guinea-pig vas deferens. Pflugers Arch. 1977;368:253-61.
16. Przyklenk K, Maynard M, Darling CE, Whittaker P. Pretreatment with D-myoinositol trisphosphate reduces infarct size in rabbit hearts: role of inositol trisphosphate receptors and gap junctions in triggering protection. J Pharmacol Exp Ther. 2005;314:1386-92.

17. Atef A, Francois $P$, Christian V, Marc-Andre $S$. The potential role of gap junction communication between cumulus cells and bovine oocytes during in vitro maturation. Mol Reprod Dev. 2005;71:358-67.

18. Weber GM, Sullivan CV. Insulin-like growth factor-I induces oocyte maturational competence but not meiotic resumption in white bass (Morone chrysops) follicles in vitro: evidence for rapid evolution of insulin-like growth factor action. Biol Reprod 2005;72:1177-86.

19. Sneddon P, Westfall DP, Colby J, Fedan JS. A pharmacological investigation of the biphasic nature of the contractile response of rabbit and rat vas deferens to field stimulation. Life Sci. 1984;35:1903-12.

20. Sneddon P, Westfall DP. Pharmacological evidence that adenosine triphosphate and noradrenaline are co-transmitters in the guinea-pig vas deferens. J Physiol. 1984;347:561-80.

21. Boselli C, Grana E. Differential effects of drugs interacting with autonomic transmitters on responses of rat vas deferens to field stimulation. J Auton Pharmacol. 2000; 20:87-97.

22. Goncalves J, Driessen B, von Kugelgen I, Starke K. Comparison of corelease of noradrenaline and ATP evoked by hypogastric nerve stimulation and field stimulation in guinea-pig vas deferens. Naunyn Schmiedebergs Arch Pharmacol. 1995;352:229-35.

23. Bader $P$, Weingart R. Conductive and kinetic properties of connexin45 hemichannels expressed in transfected HeLa cells. J Membr Biol. 2004;199:143-54.

24. Holman ME, Taylor GS, Tomita T. Some properties of the smooth muscle of mouse vas deferens. J Physiol. 1977;266:751-64.

25. Beauchamp P, Choby C, Desplantez T, de Peyer K, Green K, Yamada KA, Weingart R, Saffitz JE, Kleber AG. Electrical propagation in synthetic ventricular myocyte strands from germline connexin43 knockout mice. Circ Res. 2004;95:170-78.

26. Christ GJ. Modulation of $\alpha_{1}$-adrenergic contractility in isolated vascular tissues by heptanol: A functional demonstration of the potential importance of intercellular communication to vascular response generation. Life Sci. 1995;56:709-21.

27. Cunnane TC, Stjarne L. Frequency dependent intermittency and ionic basis of impulse conduction in postganglionic sympathetic fibres of guinea-pig vas deferens. Neurosci. 1984;11:211-29.

28. Brain KL, Jackson VM, Trout SJ, Cunnane TC. Intermittent ATP release from nerve terminals elicits focal smooth muscle $\mathrm{Ca}^{2+}$ transients in mouse vas deferens. J Physiol. 2002;15:849-62.

29. Brock JA, Cunnane TC. Characteristic features of transmitter release from sympathetic nerve terminals. Blood Vessels. 1987;24:253-60.

30. Bennett MR, Gibson WG. On the contribution of quantal secretion from closecontact and loose-contact varicosities to the synaptic potentials in the vas deferens. Philos Trans R Soc Lond B Biol Sci. 1995;347:187-204.

31. Bennett MR. Autonomic neuromuscular transmission at a varicosity. Prog Neurobiol. 1996;50:505-32.

32. Ventura $S$, Burnstock $G$. Variation in nitric oxide synthase-immunoreactive nerve fibres with age and along the length of the vas deferens in the rat. Cell Tissue Res. 1996;285:427-34.

33. Nelson WL, Makielski JC. Block of sodium current by heptanol in voltageclamped canine cardiac Purkinje cells. Circ Res. 1991;68:977-83.

34. Perez-Armendariz M, Roy C, Spray DC, Bennett MVL. Biophysical properties of gap junctions between freshly dispersed pairs of mouse pancreatic beta cells. Biophys J. 1991;59:76-92.

35. Squires PE, Hauge-Evans AC, Persaud SJ, Jones PM. Synchronization of $\mathrm{Ca}^{2+}$ signals within insulin-secreting pseudoislets: effects of gap-junctional uncouplers. Cell Calcium. 2000;27:287-96.

36. Keevil VL, Huang CL, Chau PL, Sayeed RA, Vandenberg JI. The effect of heptanol on the electrical and contractile function of the isolated, perfused rabbit heart. Pflugers Arch. 42000;40:275-82.

37. Christ GJ, Spray DC, El-Sabban M, Moore LK, Brink PR. Gap junctions in vascular tissues: Evaluating the role of intercellular communication in the modulation of vasomotor tone. Circ Res. 1996;79:631-46.

38. Christ GJ, Spektor M, Brink PR, Barr L. Further evidence for the selective disruption of intercellular communication by heptanol. Am J Physiol. 276: 1999;H1911-7.

39. Saez JC, Connor JA, Spray DC, Bennett MVL. Hepatocyte gap junctions are permeable to the second messengers, inositol 1,4,5-triphosphate, and to 


\section{PALANI and R. MANCHANDRA}

calcium ions. Proc Natl Acad Sci USA. 1989;86:2708-12.

40. Manchanda R, Venkateswarlu K. Effect of heptanol on electrical activity in the guinea-pig vas deferens. Br J Pharmacol. 1997;120:367-70.

41. Kamimura N, Suga S, Nakano K, Kanno T, Takeo T, Wakui M. Protein kinase C- dependent inhibition of $\mathrm{K}^{+}$currents in noradrenaline-induced depolarization of smooth muscle of guinea-pig vas deferens. Exp Physiol. 2000;85:37-42.

42. Mekata F. Electrical current-induced contraction in the smooth muscle of the rabbit aorta. J Physiol. 1981;317:149-61. 